Advances in Theoretical and Applied Mathematics

ISSN 0973-4554 Volume 6, Number 3 (2011), pp. 363-370

(C) Research India Publications

https://dx.doi.org/10.37622/ATAM/6.3.2011.363-370

\title{
Fuzzy Graph Structures: A Generalised Approach
}

\author{
Dinesh T. and Ramakrishnan T.V. \\ Department of Mathematical Sciences, \\ Kannur University, Mangattuparamba \\ Kannur University Campus P.O.-670 567, Kerala, India
}

\begin{abstract}
The present authors introduced the concept of a fuzzy graph structure in an earlier paper On Generalised Fuzzy Graph Structures, Appl. Math. Sc., 5(4), 2011, 173-180. Here it is further generalised by defining $\rho_{i_{1} i_{2} \ldots i_{r}}$-path, $\rho_{i_{1} i_{2} \ldots i_{r}}$ -cycle, $\rho_{i_{1} i_{2} \ldots i_{r}}$-tree, $\rho_{i_{1} i_{2} \ldots i_{r}}$-forest, fuzzy $\rho_{i_{1} i_{2} \ldots i_{r}}$-cycle, fuzzy $\rho_{i_{1} i_{2} \ldots i_{r}}$-tree and fuzzy $\rho_{i_{1} i_{2} \ldots i_{r}}$-forest. Some results are obtained.
\end{abstract}

Subject Classification: 05C72, 05C05, 05C38, 05C40

Keywords: $\rho_{i_{1} i_{2} \ldots i_{r}}$ path, $\rho_{i_{1} i_{2} \ldots i_{r}}$-connectedness, fuzzy $\rho_{i_{1} i_{2} \ldots i_{r}}$-cycle, fuzzy $\rho_{i_{1} i_{2} \ldots i_{r}}$-tree.

\subsection{Introduction}

The concept of fuzzy sets introduced by L.A. Zadeh in 1965 was used by A. Rosenfeld in 1975 [1] for defining fuzzy graph. E. Sampathkumar introduced the concept of graph structure in [2]. It is in particular, a generalisation of the notions like graphs, signed graphs and edge - coloured graphs with the colourings. He defined a graph structure $G$ as $G=\left(V, R_{1}, R_{2}, \ldots, R_{k}\right)$ where $V$ is a non-empty set and $R_{1}, R_{2}, \ldots$, $R_{k}$ are relations on $V$ which are mutually disjoint such that each $R_{i}, i=1,2, \ldots, k$ is symmetric and irreflexive.

Based on these concepts, we introduced the concept of fuzzy graph structure in [3]. Therein we defined $\rho_{i}$-edge, $\rho_{i}$-cycle, $\rho_{i}$-tree, $\rho_{i}$-forest, fuzzy $\rho_{i}$-cycle, fuzzy $\rho_{i^{-}}$ tree, fuzzy $\rho_{i}$-forest etc. and proved some results.

In this paper, we generalise the above notions and introduce some new concepts like $\rho_{i_{1} i_{2} \ldots i_{r}}$-cycle, $\rho_{i_{1} i_{2} \ldots i_{r}}$-tree, $\rho_{i_{1} i_{2} \ldots i_{r}}$-forest, fuzzy $\rho_{i_{1} i_{2} \ldots i_{r}}$-cycle, fuzzy $\rho_{i_{1} i_{2} \ldots i_{r}}{ }^{-}$ tree and fuzzy $\rho_{i_{1} i_{2} \ldots i_{r}}$-forest. 
Essential preliminaries are given wherever necessary. For more details on Graph Theory, reference may be made to [4], on Fuzzy Graph Theory, to [5] and for Graph Structures, to [2].

\subsection{Preliminaries}

We first recall the definition of fuzzy graph structure [3].

\section{Definition 2.1 [3]}

Let $G=\left(V, R_{1}, R_{2}, \ldots, R_{k}\right)$ be a graph structure and $\mu, \rho_{1}, \rho_{2}, \ldots, \rho_{k}$ be fuzzy subsets of $V, R_{1}, R_{2}, \ldots, R_{k}$ respectively such that

$\rho_{i}(x, y) \leq \mu(x) \wedge \mu(y) \forall x, y \in V, i=1,2, \ldots, k$. Then $\tilde{G}=\left(\mu, \rho_{1}, \rho_{2}, \ldots, \rho_{k}\right)$ is a fuzzy graph structure of $G$.

\section{Convention}

Throughout this paper, $G$ and $\widetilde{G}$ will have the above meaning, unless otherwise stated.

The concepts of partial fuzzy subgraph structure, $\rho_{i}$-edge, $\rho_{i}$-path, $\rho_{i}$-cycle, fuzzy $\rho_{i^{-}}$ cycle, $\rho_{i}$-forest, $\rho_{i}$-tree, fuzzy $\rho_{i}$-forest, fuzzy $\rho_{i}$-tree etc. were introduced in [3] as follows.

\section{Definition 2.2 [3]}

$\tilde{F}=\left(\mu, \tau_{1}, \tau_{2}, \ldots, \tau_{k}\right)$ is a partial fuzzy spanning subgraph structure of $\tilde{G}=\left(\mu, \rho_{1}, \rho_{2}, \ldots, \rho_{k}\right)$ if $\tau_{r} \subseteq \rho_{r}$ for $r=1,2, \ldots, k$.

\section{Definition 2.3 [3]}

Let $G$ be a graph structure and $\tilde{G}$ be a fuzzy graph structure of $G$. If $(x, y) \in \operatorname{supp}\left(\rho_{i}\right)$, then $(x, y)$ is said to be a $\rho_{i}$-edge of $\tilde{G}$.

\section{Definition 2.4 [3]}

A $\rho_{i}$-path of a fuzzy graph structure $\tilde{G}$ is a sequence of vertices, $x_{0}, x_{1}, \ldots, x_{n}$ which are distinct (except possibly $x_{0}=x_{n}$ such that $\left(x_{j-1}, x_{j}\right)$ is a $\rho_{i}$-edge for all $j=1,2, \ldots$, $n$.

\section{Definition 2.5 [3]}

$\tilde{G}$ is a $\rho_{i}$-cycle iff $\left(\operatorname{supp}(\mu), \operatorname{supp}\left(\rho_{1}\right), \operatorname{supp}\left(\rho_{2}\right), \ldots, \operatorname{supp}\left(\rho_{k}\right)\right)$ is an $R_{i}$-cycle (where an $R_{i}$-cycle is a sequence of vertices $x_{0}, x_{1}, \ldots, x_{n-1}, x_{n}=x_{0}$ in $V$ such that each $\left(x_{j-1}, x_{j}\right)$ is an $R_{i}$-edge for $j=1,2, \ldots, n$.

\section{Definition 2.6 [3]}

$\tilde{G}$ is a fuzzy $\rho_{i}$-cycle iff $\left(\operatorname{supp}(\mu), \operatorname{supp}\left(\rho_{1}\right), \operatorname{supp}\left(\rho_{2}\right), \ldots, \operatorname{supp}\left(\rho_{k}\right)\right)$ is an $R_{i}$-cycle and there exists no unique $(x, y)$ in $\operatorname{supp}\left(\rho_{i}\right)$ such that 
$\rho_{i}(x, y)=\wedge\left\{\rho_{i}(u, v) \mid(u, v) \in \operatorname{supp}\left(\rho_{i}\right)\right\}$.

\section{Definition 2.7 [3]}

$\tilde{G}$ is a $\rho_{i}$-forest if its $\rho_{i}$-edges form an $R_{i}$-forest(where an $R_{i}$-forest is a fuzzy graph structure which does not contain $R_{i}$-cycles).

\section{Definition 2.8 [3]}

$\tilde{G}$ is a $\rho_{i}$-tree if it is a $\rho_{i}$-connected $\rho_{i}$-forest.

\section{Definition 2.9 [3]}

$\tilde{G}$ is a fuzzy $\rho_{i}$-forest if it has a partial fuzzy spanning subgraph structure $\widetilde{F}_{l}=\left(\mu, \tau_{1}, \tau_{2}, \ldots, \tau_{k}\right)$ which is a $\tau_{i}$-forest where for all $\rho_{i}$-edges not in $\widetilde{F}_{l}$,

$$
\rho_{i}(x, y)<\tau_{i}^{\infty}(x, y) \text {. }
$$

\section{Definition 2.10 [3]}

$\tilde{G}$ is a fuzzy $\rho_{i}$-tree if it has a partial fuzzy spanning subgraph structure $\widetilde{F}_{l}=\left(\mu, \tau_{1}, \tau_{2}, \ldots, \tau_{k}\right)$ which is a $\tau_{i}$-tree where for all $\rho_{i}$-edges not in $\widetilde{F}_{l}, \rho_{i}(x, y)<\tau_{i}^{\infty}(x, y)$.

\subsection{Generalisation}

Now we introduce some new terms by generalising the above concepts. First we define $\rho_{i_{1} i_{2} \ldots i_{r}}$-path and $\rho_{i_{1} i_{2} \ldots i_{r}}$-connectedness.

\section{Definition 3.1}

Let $x_{0}, x_{1}, \ldots, x_{n}$ be a sequence of distinct vertices of $\tilde{G}$. Let $\rho_{i_{P}}\left(x_{j-1}, x_{j}\right)>0$ $\forall j=1,2, \ldots, n$ for some $p \in\{1,2, \ldots, r\}$ where $\rho_{i_{1}}, \rho_{i_{2}}, \ldots, \rho_{i_{r}}$ are some among $\rho_{1}, \rho_{2}$, $\ldots, \rho_{k}$. Then $x_{0}, x_{1}, \ldots, x_{n-1}, x_{n}$ is a $\rho_{i_{1} i_{2} \ldots i_{r}}$-path.

\section{Definition 3.2}

Two vertices of a fuzzy graph structure $\tilde{G}$ joined by a $\rho_{i_{1} i_{2} \ldots i_{r}}$-path are said to be $\rho_{i_{1} i_{2} \ldots i_{r}}{ }^{- \text {connected. }}$

\section{Definition 3.3}

The strength of a $\rho_{i_{1} i_{2} \ldots i_{r}}$-path $x_{0}, x_{1}, \ldots, x_{n-1}, x_{n}$ of a fuzzy graph structure $\tilde{G}$ is $\bigwedge_{j=1}^{n} \mathrm{\vee}_{q=1}^{r} \rho_{i_{q}}\left(x_{j-1}, x_{j}\right)$.

We may denote the strength of a $\rho_{i_{1} i_{2} \ldots i_{r}}$-path from $x$ to $y$ as $\rho_{i_{1} i_{2} \ldots i_{r}}(x, y)$ and the strength of a strongest $\rho_{i_{1} i_{2} \ldots i_{r}}$-path from $x$ to $y$ as $\rho_{i_{\infty}}(x, y)$.

We now define $\rho_{i_{1} i_{2} \ldots i_{r}}$-cycle, $\rho_{i_{1} i_{2} \ldots i_{r}}$-tree, $\rho_{i_{1} i_{2} \ldots i_{r}}$-forest etc.

\section{Definition 3.4}

A sequence of vertices $x_{0}, x_{1}, \ldots, x_{n-1}, x_{n}=x_{0}$ of $V$ of a fuzzy graph structure $\tilde{G}$ such 
that each $\left(x_{j-1}, x_{j}\right), j=1.2, \ldots, n$, is an $R_{i}$-edge for some $i \in\{1,2, \ldots, r\}$, is said to be an $R_{i_{1} i_{2} \ldots i_{r}}$-cycle if $R_{i_{1}}, R_{i_{2}}, \ldots, R_{i_{r}}$ are some among $R_{1}, R_{2}, \ldots, R_{k}$ which are represented in it by nonzero $R_{i}$-edges, $i=1,2, \ldots, k$.

Note that $R_{i}$-cycle [2] is an $R_{i_{1} i_{2} \ldots i_{r}}$-cycle with $i_{1}=i_{2}=\ldots=i_{r}=\mathrm{i}$.

\section{Definition 3.5}

$\tilde{G}$ is a $\rho_{i_{1} i_{2} \ldots i_{r}}$-cycle iff $\left(\operatorname{supp}(\mu), \operatorname{supp}\left(\rho_{1}\right), \operatorname{supp}\left(\rho_{2}\right), \ldots, \operatorname{supp}\left(\rho_{k}\right)\right)$ is an $R_{i_{1} i_{2} \ldots i_{r}}$-cycle and $\rho_{i_{1}}, \rho_{i_{2}}, \ldots, \rho_{i_{r}}$ are some among $\rho_{1}, \rho_{2}, \ldots, \rho_{k}$ which correspond to $R_{i_{1}}, R_{i_{2}}, \ldots, R_{i_{r}}$.

Note that $\rho_{i_{1} i_{2} \ldots i_{r}}$-cycle is a $\rho_{i}$-cycle [3] for $i_{1}=i_{2}=\ldots=i_{r}=\mathrm{i}$.

\section{Definition 3.6}

$\tilde{G}$ is a fuzzy $\rho_{i_{1} i_{2} \ldots i_{r}}$-cycle iff $\left(\operatorname{supp}(\mu), \operatorname{supp}\left(\rho_{1}\right), \operatorname{supp}\left(\rho_{2}\right), \ldots, \operatorname{supp}\left(\rho_{k}\right)\right)$ is an $R_{i_{1} i_{2} \ldots i_{r}}$-cycle and there exists no unique $(x, y)$ in $\bigcup_{q=1}^{r} \operatorname{supp}\left(\rho_{i_{q}}\right)$ such that $\mathrm{V}_{q=1}^{r} \rho_{i_{q}}(x, y)=\Lambda\left\{\mathrm{V}_{q=1}^{r} \rho_{i_{q}}(u, v) \mid(u, v) \in \mathrm{U}_{q=1}^{r} \operatorname{supp}\left(\rho_{i_{q}}\right)\right\}$

\section{Definition 3.7}

A graph structure which does not contain $R_{i_{1} i_{2} \ldots i_{r}}$-cycles for $i_{1}, i_{2}, \ldots, i_{r} \in\{1,2, \ldots, k\}$ which need not be distinct, is an $R_{i_{1} i_{2} \ldots i_{r}}$-forest.

\section{Definition 3.8}

An $R_{i_{1} i_{2} \ldots i_{r}}$-forest is an $R_{i_{1} i_{2} \ldots i_{r}}$-tree if it is connected by a $\rho_{i_{1} i_{2} \ldots i_{r}}$-path.

\section{Definition 3.9}

$\tilde{G}$ is a $\rho_{i_{1} i_{2} \ldots i_{r}}$-forest if its $\rho_{i_{p}}$-edges $(p \in\{1,2, \ldots, r\})$ form an $R_{i_{1} i_{2} \ldots i_{r}}$-forest.

\section{Definition 3.10}

$\tilde{G}$ is a $\rho_{i_{1} i_{2} \ldots i_{r}}$-tree if it is connected by a $\rho_{i_{1} i_{2} \ldots i_{r}}$-path and it is a $\rho_{i_{1} i_{2} \ldots i_{r}}$-forest.

\section{Definition 3.11}

$\tilde{G}$ is a fuzzy $\rho_{i_{1} i_{2} \ldots i_{r}}$-forest if it has a partial fuzzy spanning subgraph structure $\tilde{F}=\left(\mu, \tau_{1}, \tau_{2}, \ldots, \tau_{k}\right)$ which is a $\tau_{i_{1} i_{2} \ldots i_{r}}$-forest and $\tau_{i_{1}}, \tau_{i_{2}} \ldots, \tau_{i_{r}}$ are some among $\tau_{1}, \tau_{2}, \ldots, \tau_{k}$ which are represented in $\widetilde{F}$ where for all $\rho_{i_{p}}$-edges $(\mathrm{p} \in\{1,2, \ldots, \mathrm{r}\})$ not in $\tilde{F}, \rho_{i_{p}}(x, y)<\tau_{i \infty}(x, y)$.

\section{Definition 3.12}

$\tilde{G}$ is a fuzzy $\rho_{i_{1} i_{2} \ldots i_{r}}$-tree if it has a partial fuzzy spanning subgraph structure $\tilde{F}=\left(\mu, \tau_{1}, \tau_{2}, \ldots, \tau_{k}\right)$ which is a $\tau_{i_{1} i_{2} \ldots i_{r}}$-tree where for all $\rho_{i_{p}}{ }^{-}$ edges $(p \in\{1,2, \ldots, r\})$ not in $\tilde{F}, \rho_{i_{p}}(x, y)<\tau_{\mathbf{i} \infty}(x, y)$. 


\subsection{Some results on fuzzy $\rho_{\mathbf{i}_{1} i_{2} \ldots \mathrm{i}_{\mathbf{r}}}$-trees and fuzzy $\rho_{\mathrm{i}_{1} \mathbf{i}_{2} \ldots \mathrm{i}_{\mathbf{r}}}$-forests}

We generalise some results discussed in [3] to fuzzy $\rho_{i_{1} i_{2} \ldots i_{r}}$-trees and fuzzy $\rho_{i_{1} i_{2} \ldots i_{r}}{ }^{-}$ forests.

\section{Theorem 4.1}

$\tilde{G}$ is a fuzzy $\rho_{i_{1} i_{2} \ldots i_{r}}$-forest iff in any $\rho_{i_{1} i_{2} \ldots i_{r}}$-cycle, there exists some $\rho_{i_{p}}$-edges $(p \in\{1,2, \ldots, r\})$ such that $\rho_{i_{p}}(x, y)<\rho_{i_{\infty}}^{\prime}(x, y)$ where $\left(\mu, \rho_{1}{ }^{\prime}, \rho_{2}{ }^{\prime}, \ldots, \rho_{k}{ }^{\prime}\right)$ is the partial fuzzy spanning subgraph structure obtained by deleting $(x, y)$ and $\rho_{i_{1}}{ }^{\prime}, \rho_{i_{2}}{ }^{\prime}, \ldots, \rho_{i_{r}}{ }^{\prime}$ are defined accordingly.

\section{Proof}

\section{Sufficiency}

If $\tilde{G}$ does not contain a $\rho_{i_{1} i_{2} \ldots i_{r}}$-cycle, it is a fuzzy $\rho_{i_{1} i_{2} \ldots i_{r}}$-forest. Then there is nothing to prove.

Let $\tilde{G}$ contains a $\rho_{i_{1} i_{2} \ldots i_{r}}$-cycle. Consider some $\rho_{i_{p}}$-edge $(p \in\{1,2, \ldots, r\})(x, y)$ with $\rho_{i_{p}}(x, y)<\rho_{i_{\infty}}^{\prime}(x, y)$.

Remove $(x, y)$. Still there may be $\rho_{i_{1} i_{2} \ldots i_{r}}$-cycles. Repeat the process with some $\rho_{i_{q}}$-edge $(q \in\{1,2, \ldots, r\}), q$ need not be different from $p$.

Strength of the deleted $\rho_{i_{p}}$-edges $(p \in\{1,2, \ldots, r\})$ increases in each step. When the fuzzy graph structure is cleared of all $\rho_{i_{1} i_{2} \ldots i_{r}}$-cycles, the resultant partial fuzzy spanning subgraph structure is a $\rho_{i_{1} i_{2} \ldots i_{r}}$-forest. Let it be $\tilde{F}$.

If $(x, y) \notin \tilde{F}$, it was deleted. So there exists a $\rho_{i_{1} i_{2} \ldots i_{r}}$-path from $x$ to $y$ in $\tilde{F}$, stronger than $(x, y)$. There will be stronger $\rho_{i_{1} i_{2} \ldots i_{r}}$-paths for diverting around deleted $\rho_{i_{p}}$-edges $(p \in\{1,2, \ldots, r\})$. Repeating the process, we get a $\rho_{i_{1} i_{2} \ldots i_{r}}$-path consisting only of $\rho_{i_{p}}$-edges $(p \in\{1,2, \ldots, r\})$. Hence $\tilde{G}$ is a fuzzy $\rho_{i_{1} i_{2} \ldots i_{r}}$-forest.

\section{Necessity}

Let $\tilde{G}$ be a fuzzy $\rho_{i_{1} i_{2} \ldots i_{r}}$-forest. Let $C$ be a $\rho_{i_{1} i_{2} \ldots i_{r}}$-cycle. Some $\rho_{i_{p}}$-edge $(p \in\{1,2, \ldots, r\})(x, y)$ of $C$ is not in $\tilde{F}=\left(\mu, \tau_{1}, \tau_{2}, \ldots, \tau_{k}\right)$, the partial fuzzy spanning subgraph structure which is a $\tau_{i_{1} i_{2} \ldots i_{r}}$-forest $\left(\tau_{i_{1}}, \tau_{i_{2}} \ldots, \tau_{i_{r}}\right.$ are some among $\tau_{1}, \tau_{2}, \ldots, \tau_{k}$ which are represented in it) and $\rho_{i_{p}}(x, y)<\tau_{i_{\infty}}(x, y)$.

But $\tau_{i_{\infty}}(x, y)<\rho_{i_{\infty}}^{\prime}(x, y)$ where $\left(\mu, \rho_{1}{ }^{\prime}, \rho_{2}{ }^{\prime}, \ldots, \rho_{k}{ }^{\prime}\right)$ is obtained from $\tilde{G}$ by deleting $(x, y)$ and $\rho_{i_{1}}{ }^{\prime}, \rho_{i_{2}}{ }^{\prime}, \ldots, \rho_{i_{r}}{ }^{\prime}$ are defined accordingly.

Therefore $\rho_{i_{p}}(x, y)<\rho_{i_{\infty}}{ }^{\prime}(x, y)$.

\section{Theorem 4.2}

Let $\tilde{G}$ be a fuzzy graph structure. If there is at most one strongest $\rho_{i_{1} i_{2} \ldots i_{r}}$-path between any two vertices, then $\tilde{G}$ must be a fuzzy $\rho_{i_{1} i_{2} \ldots i_{r}}$-forest. 


\section{Proof}

Suppose there exists at most one strongest $\rho_{i_{1} i_{2} \ldots i_{r}}$-path between any two vertices of $\tilde{G}$.

If possible, let $\tilde{G}$ be not a fuzzy $\rho_{i_{1} i_{2} \ldots i_{r}}$-forest. Then by definition, there exists a $\rho_{i_{1} i_{2} \ldots i_{r}}$-cycle $C$ such that for every $\rho_{i_{p}}$-edge $(\mathrm{p} \in\{1,2, \ldots, \mathrm{r}\})(x, y)$

in $C, \rho_{i_{p}}(x, y) \geq \rho_{i_{1} i_{2} \ldots i_{r}}{ }^{\prime}(x, y)$ where $\left(\mu, \rho_{1}{ }^{\prime}, \rho_{2}{ }^{\prime}, \ldots, \rho_{k}{ }^{\prime}\right)$ is the partial fuzzy spanning subgraph structure obtained by deleting $(x, y)$ from $\tilde{G}$ and $\rho_{i_{1} i_{2} \ldots i_{r}}{ }^{\prime}(x, y)$ is the strength of $\rho_{i_{1} i_{2} \ldots i_{r}}$-path (there is only one such $\rho_{i_{1} i_{2} \ldots i_{r}}$-path) from $x$ to $y$ not involving $(x, y)$. ie., $(x, y)$ is the strongest $\rho_{i_{1} i_{2} \ldots i_{r}}$-path from $x$ to $y$.

If $(x, y)$ is the weakest $\rho_{i_{p}}$-edge $(p \in\{1,2, \ldots, r\})$ of $C$, the remaining $\rho_{i_{p}}$-edges $(p \in\{1,2, \ldots, r\})$ of $C$ form a strongest $\rho_{i_{1} i_{2} \ldots i_{r}}$-path which is a contradiction.

Therefore $\tilde{G}$ is a fuzzy $\rho_{i_{1} i_{2} \ldots i_{r}}$-forest.

\section{Theorem 4.3}

Let $\tilde{G}$ be a fuzzy $\rho_{i_{1} i_{2} \ldots i_{r}}$-tree and $\tilde{G}^{*}=\left(\operatorname{supp}(\mu), \operatorname{supp}\left(\rho_{1}\right), \operatorname{supp}\left(\rho_{2}\right), \ldots, \operatorname{supp}\left(\rho_{k}\right)\right)$ be not a $\rho_{i_{1} i_{2} \ldots i_{r}}$-tree. Then there exists at least one $\rho_{i_{p}}$-edge $(p \in\{1,2, \ldots, r\})(u, v)$ in $\bigcup_{q=1}^{r} \operatorname{supp}\left(\rho_{i_{q}}\right)$ for which $\rho_{i_{p}}(u, v)<\rho_{i_{\infty}}(u, v)$.

\section{Proof}

Let $\tilde{G}$ be a fuzzy $\rho_{i_{1} i_{2} \ldots i_{r}}$-tree. Then there exists a partial fuzzy spanning subgraph structure $\tilde{F}=\left(\mu, \tau_{1}, \tau_{2}, \ldots, \tau_{k}\right)$ which is a $\tau_{i_{1} i_{2} \ldots i_{r}}$-tree and for every $\rho_{i_{p}}$ -edge $(p \in\{1,2, \ldots, r\})(u, v) \notin \widetilde{F}, \rho_{i_{p}}(u, v)<\tau_{i_{\infty}}(u, v)$.

Also $\tau_{i_{\infty}}(u, v) \leq \rho_{i_{\infty}}(u, v)$.

Therefore $\rho_{i_{p}}(u, v)<\rho_{i_{\infty}}(u, v) \forall \rho_{i_{p}}$-edge $(u, v) \notin \tilde{F}$. $\tilde{G}$ is a fuzzy $\rho_{i_{1} i_{2} \ldots i_{r}}$-tree and $\tilde{G}^{*}$ is not a $\rho_{i_{1} i_{2} \ldots i_{r}}$-tree.

Therefore there exists at least one $\rho_{i_{p}}$-edge $(p \in\{1,2, \ldots, r\}) \notin \tilde{F}$. ie., there exists at least one $\rho_{i_{p}}$-edge $(p \in\{1,2, \ldots, r\})(u, v)$ in $\bigcup_{q=1}^{r} \operatorname{supp}\left(\rho_{i_{q}}\right)$ with $\rho_{i_{p}}(u, v)<\rho_{i_{\infty}}(u, v)$.

\section{Lemma 1}

Let $\tilde{G}$ be a fuzzy graph structure with $\rho_{i_{p}}(u, v)=\mu(u) \wedge \mu(v)$ for some $p \in\{1,2, \ldots, r\}$ and for every $\rho_{i_{p}}$-edge $(u, v) \in \bigcup_{q=1}^{r} \operatorname{supp}\left(\rho_{i_{q}}\right)$ where $\bigcup_{q=1}^{r} \operatorname{supp}\left(\rho_{i_{q}}\right) \neq \phi$. Then $\rho_{i_{\infty}}(u, v)=\rho_{i_{p}}(u, v)$ for that $p$.

\section{Proof}

$\rho_{i_{p}}(u, v)=\mu(u) \wedge \mu(v)$ for every $\rho_{i_{p}}$-edge $(u, v)$ for some $p \in\{1,2, \ldots, r\},(u, v) \in$ $\bigcup_{q=1}^{r} \operatorname{supp}\left(\rho_{i_{q}}\right) . \rho_{i_{\infty}}(u, v)=$ strength of the strongest $\rho_{i_{1} i_{2} \ldots i_{r}}$-path from $u$ to $v$. ie., $\rho_{i_{\infty}}(u, v)=\mu(u) \wedge \mu(v)=\rho_{i_{p}}(u, v)$ for that $p$. 


\section{Theorem 4.4}

Let $\tilde{G}$ be a fuzzy $\rho_{i_{1} i_{2} \ldots i_{r}}$-tree. Then $\rho_{i_{p}}(u, v)<\mu(u) \wedge \mu(v)$ for some $\rho_{i_{p}}$-edge $(u$, v) $(p \in\{1,2, \ldots, r\})$, in $\bigcup_{q=1}^{r} \operatorname{supp}\left(\rho_{i_{q}}\right)$.

\section{Proof}

If possible, let $\rho_{i_{p}}(u, v)=\mu(u) \wedge \mu(v) \forall(u, v) \in \bigcup_{q=1}^{r} \operatorname{supp}\left(\rho_{i_{q}}\right)$.

Then by lemma, $\rho_{i_{p}}(u, v)=\rho_{i_{\infty}}(u, v)$ for that $p$ for which $\rho_{i_{p}}(u, v)=\mu(u) \wedge \mu(v)$.

Let $\tilde{G}$ be a fuzzy $\rho_{i_{1} i_{2} \ldots i_{r}}$-tree. Then it has a partial fuzzy spanning subgraph structure $\tilde{F}=\left(\mu, \tau_{1}, \tau_{2}, \ldots, \tau_{k}\right)$ which is a $\tau_{i_{1} i_{2} \ldots i_{r}}$-tree with $\rho_{i_{p}}(u, v)<\tau_{i_{\infty}}(u, v) \forall \rho_{i_{p}}{ }^{-}$ edge $(u, v) \notin \tilde{F}$.

Therefore $\rho_{i_{\infty}}(u, v)<\tau_{i_{\infty}}(u, v)$ which is a contradiction.

Hence $\rho_{i_{p}}(u, v)<\mu(u) \wedge \mu(v)$ for some $\rho_{i_{p}}$-edge $(p \in\{1,2, \ldots, r\})(u, v)$.

\section{Theorem 4.5}

Let $\tilde{G}$ be a $\rho_{i_{1} i_{2} \ldots i_{r}}$-cycle. $\tilde{G}$ is a fuzzy $\rho_{i_{1} i_{2} \ldots i_{r}}$-cycle iff $\tilde{G}$ is not a fuzzy $\rho_{i_{1} i_{2} \ldots i_{r}}$-tree.

\section{Proof}

Let $\tilde{G}$ be a fuzzy $\rho_{i_{1} i_{2} \ldots i_{r}}$-cycle.

If possible, let it be a fuzzy $\rho_{i_{1} i_{2} \ldots i_{r}}$-tree also. Then it has a partial fuzzy spanning subgraph structure $\tilde{F}=\left(\mu, \tau_{1}, \tau_{2}, \ldots, \tau_{k}\right)$ which is a $\tau_{i_{1} i_{2} \ldots i_{r}}$-tree. Then

$\left[\bigcup_{q=1}^{r} \operatorname{supp}\left(\rho_{i_{q}}\right)-\bigcup_{q=1}^{r} \operatorname{supp}\left(\tau_{i_{q}}\right)\right]=\{(u, v)\}$ for some $u, v \in V$ since $\tilde{G}$ is a $\rho_{i_{1} i_{2} \ldots i_{r}}$-cycle.

There does not exist a unique $(x, y)$ in $\bigcup_{q=1}^{r} \operatorname{supp}\left(\rho_{i_{q}}\right)$ such that

$$
\mathrm{V}_{q=1}^{r} \rho_{i_{q}}(x, y)=\Lambda\left\{\mathrm{V}_{q=1}^{r} \rho_{i_{q}}(u, v):(u, v) \in \mathrm{U}_{q=1}^{r} \operatorname{supp}\left(\rho_{i_{q}}\right)\right\}
$$

Therefore there exists no $\tau_{i_{1} i_{2} \ldots i_{r}}$-path in $\tilde{F}$ from $u$ to $v$ having greater strength than $\rho_{i_{1} i_{2} \ldots i_{r}}(u, v)$. Otherwise $\tilde{G}$ will not be a fuzzy $\rho_{i_{1} i_{2} \ldots i_{r}}$-cycle.

Thus $\tilde{G}$ is not a fuzzy $\rho_{i_{1} i_{2} \ldots i_{r}}$-tree.

Conversely, let $\tilde{G}$ be not a fuzzy $\rho_{i_{1} i_{2} \ldots i_{r}}$-tree. Then it has no partial fuzzy spanning subgraph structure $\tilde{F}$ which is a $\tau_{i_{1} i_{2} \ldots i_{r}}$-tree.

$\tilde{G}$ is a $\rho_{i_{1} i_{2} \ldots i_{r}}$-cycle. Let $\left(\mu, \tau_{1}, \tau_{2}, \ldots, \tau_{k}\right)$ be a partial fuzzy spanning subgraph structure of $\tilde{G}$, which is a $\tau_{i_{1} i_{2} \ldots i_{r}}$-tree with $\rho_{i_{p}}(u, v)<\tau_{i_{\infty}}(u, v)$ for all $\rho_{i_{p}}$-edge

$(p \in\{1,2, \ldots, r\})(u, v) \notin \tilde{F}$.

$\tau_{i_{\infty}}(u, v) \leq \rho_{i_{p}}(u, v) \forall \rho_{i_{p}}$-edge $(u, v) \operatorname{in} \bigcup_{q=1}^{r} \operatorname{supp}\left(\rho_{i_{q}}\right), p \in\{1,2, \ldots, r\}$, where $\tau_{i_{p}}(u, v)=0 \forall p=1,2, \ldots, r$.

$\tau_{i_{p}}(x, y)=\rho_{i_{p}}(x, y) \forall(x, y) \in \bigcup_{q=1}^{r} \operatorname{supp}\left(\rho_{i_{q}}\right)-\{(u, v)\}$ for $p \in\{1,2, \ldots, r\}$. 
Therefore there does not exist unique $(x, y)$ with

$$
\mathrm{V}_{q=1}^{r} \rho_{i_{q}}(x, y)=\Lambda\left\{\bigvee_{q=1}^{r} \rho_{i_{q}}(u, v):(u, v) \in \operatorname{supp}\left(\rho_{i_{q}}\right)\right\}
$$

Therefore $\tilde{G}$ is a fuzzy $\rho_{i_{1} i_{2} \ldots i_{r}}$-cycle.

\section{References}

[1] Rosenfeld, A., 1975, Fuzzy Graphs in "Fuzzy Sets and their Applications to Cognitive and Decision Processes"(eds. L.A. Zadeh, K.S. Fu \& M. Shimura), Acad. Press, New York, pp 77-95.

[2] Sampathkumar, E., Dec 2006, Generalised Graph Structures, Bull. Kerala Math. Assoc., Vol 3, No.2, 65-123.

[3] Dinesh, T. \& Ramakrishnan, T.V., On Generalised Fuzzy Graph Structures, Appl. Math. Sc., 5(4), 2011, 173-180.

[4] Harary, F. - Graph Theory, 1995, Narosa Pub. House.

[5] Mordeson, J.N. \& Nair, P.S., 2000, Fuzzy Graphs and Fuzzy Hypergraphs, Physica- verlag. 